Instituto Internacional de Investigación y Desarrollo Tecnológico Educativo INDTEC, C.A.

DOI: https://doi.org/10.29394/Scientific.issn.2542-2987.2020.5.17.8.164-182

OAI-PMH: http://www.indteca.com/ojs/index.php/Revista Scientific/oai

Artículo Original / Original Article

\title{
La Práctica de Enfermería: desde el Contexto Hospitalario en Venezuela
}

\author{
Autor: Rubén Eliut Hernández Ortega \\ Universidad de las Ciencias de la Salud, UCS \\ rubenho1977@gmail.com \\ Caracas, Venezuela \\ https://orcid.org/0000-0003-3844-278X
}

\section{Resumen}

El presente artículo tiene como objetivo develar los elementos epistémicos de la práctica de enfermería en el contexto hospitalario venezolano, a partir de la interacción con profesionales de enfermería (método cualitativo), de los establecimientos del Sistema Público Nacional de Salud (SPNS) del Hospital Dr. Jesús Mata de Gregorio y el Hospital Dr. José María Vargas, Caracas, Venezuela. Recurriendo a la revisión de la Ley de Ejercicio Profesional de la Enfermería (2005); y el Código Deontológico (2009); con referentes del humanismo como Acosta y Acosta (2019); y el cuidado humanizado de Guerrero-Ramírez, Meneses-La Riva y De La Cruz-Ruiz (2015); sustentado en el enfoque interpretativo, paradigma cualitativo, diseño emergente de Guba y Lincoln (2000). Se utilizó la entrevista a profundidad como técnica de recolección de datos, y para el análisis, se empleó el Método Comparativo Continuo de Strauss y Corbin (2002), a través de la codificación abierta, axial y selectiva, surgiendo como categoría central, la Práctica Humanista de Enfermería. Los hallazgos reflejan que dicha práctica, se fundamenta en el humanismo, los valores éticos-morales, actos reflexivos y las competencias. Concluyendo que, el conocimiento es esencial para realizar esta práctica, ya que está ligado a la comprensión de los elementos filosóficos que rigen la conducta profesional, contribuyendo a incentivar la investigación para fortalecer el quehacer enfermero en el contexto hospitalario venezolano. enfermería.

Palabras clave: humanismo; personal paramédico; servicio de

Cómo citar este artículo:

Hernández, R. (2020). La Práctica de Enfermería: desde el Contexto Hospitalario en Venezuela. Revista Scientific, 5(17), 164-182, e-ISSN: 2542-2987. Recuperado de: https://doi.org/10.29394/Scientific.issn.2542-2987.2020.5.17.8.164-182

Fecha de Recepción: 20-01-2020
Fecha de Aceptación: 28-04-2020
Fecha de Publicación: 05-08-2020 
OAI-PMH: http://www.indteca.com/ojs/index.php/Revista_Scientific/oai

Artículo Original / Original Article

\title{
Nursing Practice: from the Hospital Context in Venezuela
}

\begin{abstract}
The objective of this article is to reveal the epistemic elements of nursing practice in the Venezuelan hospital context, based on the interaction with nursing professionals (qualitative method), of the establishments of the National Public Health System (SPNS) of Hospital Dr Jesús Mata de Gregorio and the Hospital Dr. José María Vargas, Caracas, Venezuela. Resorting to the revision of the Nursing Professional Practice Law (2005); and the Code of Ethics (2009); with references of humanism such as Acosta and Acosta (2019); and the humanized care of Guerrero-Ramírez, Meneses-La Riva and De La Cruz-Ruiz (2015); based on the interpretive approach, qualitative paradigm, emerging design of Guba and Lincoln (2000). The in-depth interview was used as a data collection technique, and for the analysis, the Continuous Comparative Method of Strauss and Corbin (2002) was used, through open, axial and selective coding, emerging as a central category, the Practice Nursing Humanist. The findings reflect that this practice is based on humanism, ethical-moral values, reflective acts and competencies. Concluding that knowledge is essential to carry out this practice, since it is linked to the understanding of the philosophical elements that govern professional conduct, helping to encourage research to strengthen nursing in the Venezuelan hospital context.
\end{abstract}

Keywords: humanism; paramedical personnel; nursing.

How to cite this article:

Hernández, R. (2020). Nursing Practice: from the Hospital Context in Venezuela. Revista

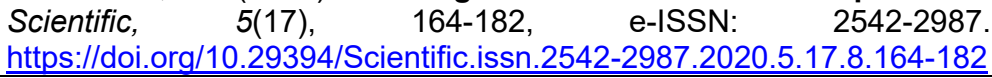

Date Received: 20-01-2020
Date Acceptance:

28-04-2020
Date Publication:

05-08-2020 


\section{Introducción}

La práctica de enfermería, está basada en el amor a la preservación de la vida y la salud, bajo una actitud reflexiva, sostenida en valores humanos, con el propósito de desarrollar y consolidar los patrones de pensamiento enfermero, reafirmar competencias y habilidades, contribuyendo así al bienestar biopsicosocial y espiritual de la persona, familia y comunidad.

Como señala la Ley de Ejercicio Profesional de la Enfermería (2005a), en el numeral 2:

La práctica de sus funciones en el cuidado... se sustenta en una relación de interacción humana y social entre el o la profesional y el o la paciente, familia y comunidad. La esencia del cuidado de enfermería está en cuidar, rehabilitar, promover la salud, prevenir enfermedades y contribuir a una vida digna de la persona (art. 2).

Por lo tanto, el estudio se realiza basado en el ser ontológico de la profesión, los aspectos éticos, bioéticos y morales, establecidos en el deber ser, y en la exteriorización del cuidado humano, como el objeto de la profesión, donde la concepción epistémica conduce al desarrollo de esta ciencia, a través de una conciencia reflexiva que permita mantener un óptimo bienestar físico, mental, social y espiritual de las personas.

El mismo se sustenta en el enfoque interpretativo-crítico, paradigma cualitativo emergente, el cual permite explicar las distintas posturas que los informantes clave tienen en relación a la práctica de enfermería, surgiendo de éstos que el cuidado es la esencia de la misma, por ende, a los profesionales de enfermería les corresponde repensarlo como un elemento filosófico de entendimiento entre teoría y práctica, donde compete una intervención basada en las necesidades humanas de las personas, reconociendo, respetando y defendiendo la conciencia de éstas, de modo que el cuidado sea un acto único y trascendente en las vidas de ambos sujetos, ya que el mismo es la razón moral de esta ciencia, no es un procedimiento o una técnica, es un proceso 
interconectado, intersubjetivo y de sentimientos compartidos.

Es por esta razón que socialmente se concibe al ejercicio enfermero, como un proceso humanista, en el cual se detecta las determinantes que condicionan el funcionamiento básico de los aspectos vitales del ser humano, logrando que éste reconozca los procesos de salud y de enfermedad en conformidad con sus modos de vida y, por ende, se hagan más autónomos en cuanto a la participación integral en el medio social donde viven. Este aspecto cobra relevancia y a su vez cumple con el encargo social establecido en la Constitución de la República Bolivariana de Venezuela (2009): en cuanto al posicionamiento de la profesión de la Enfermería en el contexto venezolano.

Por lo tanto, el beneficio radica en demostrar un nivel reflexivo que integre las funciones de enfermería (asistenciales, docentes, administrativas e investigativas), donde asuman los roles que demanda la sociedad actual y se erradique el modelo mecanicista y capitalista que ha permitido que los profesionales de la salud hayan sido víctima del concepto estratégico de entrega a la oferta y la demanda, insertados en el mercado laboral sólo con una preparación instrumental, capacitados para el ejercicio técnico y procedimental, ocasionando muchas veces la deshumanización del cuidado.

Para esta investigación, se consideró que al aportar desde la ciencia de la educación un conocimiento que intenta responder a uno de los problemas sociales del contexto venezolano, tal es el caso de la deshumanización de los cuidados de enfermería, el mismo se ofrece epistémicamente, de acuerdo con un fundamento referencial importante para el desarrollo integral de las enfermeras y los enfermeros, ya que ayuda a la reflexividad de su acción humana y contribuye a incentivar la Investigación, como parte de la transformación social del País.

Asimismo, la pertinencia radica en indagar, develar y buscar respuestas a este problema que atañe a la sociedad, construyendo un cuerpo de postulados que emerge de la interacción con los sujetos estudiados y que 
produce un referente que define dicha práctica, cónsono con el objetivo de éste, el cual se enmarca en develar los elementos epistémicos de la práctica de enfermería en el contexto hospitalario venezolano, a partir de la interacción con profesionales de enfermería, de los establecimientos del Sistema Público Nacional de Salud (SPNS) del Hospital Dr. Jesús Mata de Gregorio y el Hospital Dr. José María Vargas, Caracas, Venezuela.

\section{Metodología (Materiales y métodos)}

Se asumió el paradigma cualitativo-interpretativo, mediante el cual Guba y Lincoln (2000a), indican que: "el objetivo de la ciencia es la predicción y el control de los fenómenos naturales" (pág. 115).

Paralelamente, Guba y Lincoln (2000b), expresan que:

El comportamiento humano, a diferencia de los objetos físicos, no puede entenderse sin referencia a los significados y propósitos que los actores humanos les proporcionan a sus actividades. Se asevera que los datos cualitativos pueden proporcionar una valiosa percepción aguda o "insight" sobre el comportamiento humano (pág. 116).

Desde esta postura, la realidad está suscrita en las descripciones de las vivencias de los enfermeros y las enfermeras entrevistas. Es decir; el enfoque estudiado permitió comprender las significaciones de la práctica de enfermería, a partir de las experiencias obtenidas a lo largo de su carrera profesional, a través de la integración de sus funciones y la interconexión de elementos que favorecen el desarrollo de una práctica humanista de enfermería, con la ayuda intersubjetiva del investigador.

El diseño del estudio es emergente, debido a que éste se fue elaborando en la medida como avanzó la misma, concibiéndose como un esquema flexible y permeable, en el cual fueron emergiendo aspectos que se incorporaron, tales como referentes, escenarios de investigación, sujetos estudiados y conceptos surgidos, que focalizaron en forma interactiva las 
significaciones del fenómeno estudiado.

Como escenarios para la realización de esta investigación, se seleccionaron dos (2) establecimientos de salud del Sistema Público Nacional de Salud (SPNS), los cuales son el Hospital Psiquiátrico Dr. Jesús Mata de Gregorio y el Hospital General Dr. José María Vargas de Caracas, Venezuela, en el periodo del mes de enero del año 2018 y el mes de agosto del año 2019. En donde los Consejos Científico-académicos y los Comité de Investigación y Ética de ambas instituciones, aprobaron ejecutar el estudio en sus instalaciones y permitir la realización de las entrevistas a los actores sociales en estudio.

Los informantes clave de ésta investigación, fueron las personas que, por sus vivencias, experiencias y relaciones directas con la enfermería, conciben de forma concreta la episteme de ésta profesión, por ello, comprenden ampliamente la práctica, a partir de la conjugación teórica, sus percepciones y sus amplias trayectorias dentro del ejercicio, lo que llevó a seleccionar a cuatro (4) enfermeras y enfermeros que laboran en los escenarios antes señalados, eligiéndolos de manera intencional, cuyos criterios de selección comprenden: trayectoria profesional entre 18 y 38 años de experiencia, especialistas, magíster y doctores, el cumplimiento integral de diversos roles profesionales: supervisores de área, gerentes de enfermería, docentes de pre y postgrados y directores de docencia.

En este sentido, se establece que estos actores sociales son quienes, desde su cotidianidad, comparten la experiencia de la práctica estudiada, en el contexto hospitalario mencionado, a partir de sus diferentes perspectivas, lo que constituye una noción parcial de su realidad, siendo ésta analizada exhaustivamente por el investigador, permitiéndole construir familias de categorías, con el fin de explicar detalladamente el fenómeno d estudio.

Como técnica para recolectar la información, se utilizó la entrevista a profundidad, utilizada para interpretar la indagación alcanzada, en relación con 
la práctica del profesional de enfermería. Éstas, se iniciaron con una pregunta generadora: ¿Qué es para Ud. la práctica de enfermería? En donde el investigador fue el moderador e instrumento principal de investigación, como señalan Taylor y Bogdan (1984):

En este tipo de entrevistas el investigador es el instrumento de la investigación y no el protocolo o formulario de la entrevista. Su rol implica no sólo obtener respuestas, sino también aprender qué preguntas hacer y cómo hacerlas. Requiere de muchos encuentros con los informantes, el avance es muy lento, trata de aprender lo que es importante para los informantes antes de enfocar los intereses de la investigación. (pág. 101).

En consecuencia, para aplicar esta técnica, se realizaron encuentros dinámicos, establecidos entre los parámetros del respeto, la confianza y el profesionalismo.

Como técnicas de análisis de los datos, se utilizó el Método Comparativo Continuo, que mencionado a Strauss y Corbin (2002a): "consiste en codificar y analizar datos en forma continua para desarrollar conceptos. Realizando una comparación de incidente con incidente, identificando sus propiedades, explorando sus interrelaciones y logrando integrarlo en una teoría que guarda una lógica razonable" (pág. 80).

El método aplicado, permitió contrastar continuamente los datos obtenidos, con el fin de obtener conceptos que definan la teoría sustantiva, a través de varios procedimientos, como lo son: almacenamiento de datos, codificación de la información y notas de campo, con el fin de analizarla, interrelacionarla y compararla; logrando una saturación teórica e identificando la categoría central: La Práctica Humanista de Enfermería, lo que se evidencia como teoría sustantiva, que conforme a Strauss y Corbin (2002b): "es necesario validar o contrastar con la teoría formal" (pág. 172).

El método se desarrolló en tres (3) partes básicas, las cuales son: 1). 
Codificación abierta; 2). Axial; y 3). Selectiva. En donde la primera, consistió en colocar una etiqueta a estas ideas o estructuras conceptuales, permitiendo que se identificara y clasificara a partir de los datos obtenidos. En la segunda, se reagruparon los datos que se habían fragmentado en la codificación abierta, permitiendo relacionar categorías con subcategorías, relacionándose por medio de las definiciones e identificando de forma detallada las categorías principales. La tercera y última parte, se llevó a cabo mediante la unión ordenada de los elementos que conforman la categoría central, en este sentido, la teoría sustantiva, se explicó por medio de conceptos y categorizaciones en el fenómeno estudiado.

De igual forma se utilizó la técnica denominada, análisis de contenido, la cual consiste, según Rojas (2007): en "[...] un enfoque metodológico para el análisis sistemático de textos siguiendo ciertas reglas y pasos" (pág. 134).

En referencia a ello, no basta solamente con observar e interpretar el fenómeno social estudiado en el tiempo de su ocurrencia, por lo tanto, es importante interpretar los textos descritos como fundamentos jurídicos, que regulan y norman el ejercicio profesional de la enfermería, tales como la Ley de Ejercicio Profesional de la Enfermería (2005b); y el Código Deontológico (2009a): de los profesionales de enfermería de la República Bolivariana de Venezuela, ya que se convierte en un registro con referente socio-histórico, epistemológico y ontológico en relación a la práctica de enfermería.

Los hallazgos del proceso de la investigación surgen de los testimonios de los informantes clave, cuyos datos permitieron analizar dichas informaciones, emergiendo cuatro (4) categorías de análisis e interpretación: 1). Actitud Humana, subcategoría: Acción-reflexión-acción; 2). Práctica Humanista de Enfermería, subcategoría: Competencias profesionales; 3). Bioética, subcategoría: Principios bioéticos y Valor humano; y 4). Prosecución de la Formación Académica, subcategoría: Proceso cognoscitivo.

En este sentido, emerge que la actitud del profesional de enfermería 
está en correspondencia con el compromiso ético-moral y la responsabilidad que se tiene con el trabajo, con la profesión y con las personas que atienden o le brindan cuidados, ya que el cuidar al otro es un acto que conlleva a tener una disposición condescendiente con las personas, lo que conlleva a asumir una actitud de interacción entre humanos. Corresponde aplicar cuidados como un acto actos de amor por y hacia la humanidad, conjugando la vida por encima del usufructo o lucro.

El proceso de cuidados, depende fundamentalmente de los valores éticos y morales, como una demostración consciente que significa el acto moral de la vida y para la vida, donde el comportamiento debe guiar a un trato que depende del respeto por la dignidad, la vida, las condiciones sociales, la orientación sexual, cultural, social y étnica, los credos y las creencias, observando la naturaleza donde viven y se desarrollan como parte fundamental de sus vidas, teniendo en cuenta que tienen convicciones personales únicas.

El conocimiento es fundamental en la práctica de la enfermería, porque está ligado a la comprensión de los elementos que rigen la conducta ética del profesional, las cualidades humanas, los valores, la actitud frente al otro y las competencias profesionales. Estando inmersa la obligación de actualizar constantemente sus conocimientos, en pro de mejorar su actuación, indagando de manera permanente sobre cómo la teoría diverge o converge con la práctica, para establecer parámetros de pensamiento enfermero que ayuden a consolidar las bases fundamentales de esta ciencia.

El quehacer enfermero se fundamenta en el humanismo, como esencia plena del cuidado, evidenciándose como un sujeto que ayuda a otro consciente de lo valioso de la vida, del respeto y su dignidad en todas las etapas del desarrollo, manteniendo una disposición que ayude a la satisfacción de las necesidades de sus semejantes, en el restablecimiento de la salud y el bienestar de las personas. 
Y finalmente, surge que el quehacer enfermero, está fundamentado en la adquisición y desarrollo de conocimientos, habilidades y capacidades que son expresadas en la aplicación y demostración de competencias profesionales, que están inmersas cognitivamente en el perfil o función que realiza.

\section{Resultados (análisis e interpretación de los resultados)}

Las categorías se interrelacionaron intrínsecamente con categorías, luego con las subcategorías y, éstas a su vez con las demás subcategorías, surgiendo como categoría central: Práctica Humanista de Enfermería, que sirvió para contrastar dicha teoría, a partir del humanismo y el cuidado humano.

En tal sentido se asume que la enfermería es una profesión humanista, que vela por el bienestar físico, mental, espiritual y social de las personas que cuidan diariamente, conllevando a un quehacer, que se asume a partir del cuidado humano que brinda este profesional, por medio del conocimiento técnico-científico, basado en competencias, destrezas y habilidades propias para brindar una atención humanizada.

En términos concisos se refleja que la práctica de enfermería, se centra en las competencias profesionales, las cuales son vistas desde la adquisición de capacidades y saberes, que distinguen la acción realizada por los enfermeros y las enfermeras de los contextos hospitalarios estudiados, por ende, concibe al hombre como un ser que está compuesto por aspectos físico, mental, espiritual y social, producto de su estructura biológica, imperando el respeto a la vida y la dignidad de las personas, ya que son la razón de ser del proceso de cuidado, por lo tanto, el mismo pierde su sentido si no existe un interés fundamentado en la ayuda humanitaria hacia ésta, para lograr la satisfacción de las necesidades reales y potenciales que están interfiriendo su funcionamiento básico, comprendiendo y aplicando el humanismo, como 
tendencia propia del reencuentro del hombre con lo humano.

En este sentido, se asumió la postura esgrimida por el maestro Prieto (1959), citado por Acosta y Acosta (2019a): "la formación educativa de los ciudadanos se realiza atendiendo a los requerimientos que el desarrollo económico y social demanda (...) son los hombres formados los que generan desarrollo y riqueza (...)" (pág. 354). Considera Acosta y Acosta (2019b): que "la sociedad requiere de ciudadanos que sean capaces de contribuir al desarrollo del país, no de manera individualizada, sino para el beneficio del colectivo" (pág. 354).

Es por ello que el motivo principal de la corriente filosófica de Prieto Figueroa, fue la capacitación de un ser integral, enfocándose en una formación con principios para la vida, un proceso de enseñanza que logre un saber productivo, vinculándose a su realidad y contexto social, centre su desempeño en las personas, como seres con aspectos: biológico, psicológico, espiritual y social, por ende, el humanismo es la esencia filosófica de la práctica de enfermería, donde, la manera de comunicarse, la actitud, la expresión verbal y gestual, transmite la unidad existente entre el cuerpo, la mente y el espíritu humano, interconectándose al medio ambiente, los valores humanos y el respeto hacia las opciones y decisiones que toman las personas. La importancia de ello radica en cuidar la vida y la salud, desde un fundamento humano, cuya intención consciente sea realizar una práctica a partir del cuidado trasformador.

Bajo estas mismas perspectivas, Watson (1988), citado por GuerreroRamírez, Meneses-La Riva y De La Cruz-Ruiz (2015a), considera que la práctica de enfermería se sustenta en el cuidado transpersonal como una forma singular que aborda el profesional de enfermería, en ese sentido refiere que: "es una clase especial de cuidado humano que depende del compromiso moral de la enfermera, de proteger y realzar la dignidad humana; permitiendo satisfacer las necesidades básica de los pacientes" (pág. 139). 
En efecto, Watson (1988), citado por Guerrero-Ramírez, Meneses-La Riva y De La Cruz-Ruiz (2015b), sostienen que:

La Teoría del Cuidado Humano se basa en la armonía entre mente, cuerpo y alma, a través de una relación de ayuda y confianza entre la persona cuidada y el cuidador. Su teoría tiene un enfoque filosófico (existencial-fenomenológico), con base espiritual, cuidado como un ideal moral, y ético de la enfermería. Filosofía de Jean Watson sostiene que el Cuidado Humano comprende; un Compromiso Moral (Proteger y realzar la dignidad humana; va más allá de una evaluación médica), la experiencia, percepción y la conexión: (Mostrando interés profundo a la persona) (pág. 137).

A partir de la identificación de que concibe la esencia de la enfermería, surgen una serie de elementos que los conduce a realizar una práctica humanista, en donde el conocimiento se enlaza con los preceptos establecidos en el Código Deontológico (2009b): de los profesionales de enfermería de la República Bolivariana de Venezuela, en sus artículos N. ${ }^{\circ} 2$ y N. ${ }^{\circ} 3$ y en la Ley de Ejercicio Profesional de la Enfermería (2005c): en sus artículos 2 y 13. Los cuales norman la actuación de estos profesionales a partir de una relación humana, por lo tanto, el conocimiento constituye la guía para la realización de los cuidados, valiéndose de técnicas y la adquisición de habilidades, que nutren el proceso de atención de enfermería.

Sin embargo, en las visitas, las observaciones y las notas de campo realizadas en el contexto estudiado, aunado a diversas conversaciones con los entrevistados, se pudo descubrir la existencia de un grupo de profesionales, que presentan una actuación competitiva limitada, trayendo como resultado la realización de una práctica distante de la naturaleza del arte de cuidar, considerándose que la misma está condicionada por factores externos como la desmejora de sueldos, salarios y las múltiples tareas delegadas.

El déficit de este importante talento humano y la falta de supervisión en 
cuanto a la aplicación de normas, además de los factores internos producto de la apatía, la desmotivación, la falta de sensibilidad, la aplicación de contravalores, el irrespeto a las normas éticas y la separación entre práctica e investigación, reflejada en la desactualización o poca iniciativa de proseguir estudios, lo que hace pensar que éstas actitudes, han hecho aparecer conductas que disminuye la calidad de atención y el cuidado de enfermería, como lo plantean Terán y Rico (2018):

[...] En el caso del profesional de enfermería ha existido una deficiencia irrevocable en el desarrollo de estos valores como la ética, la moral, igualdad, solidaridad, respeto, responsabilidad, entre otros. $Y$ estas circunstancias se evidencian al momento de su desempeño laboral tanto en centros asistenciales públicos como privados [...] (pág. 65).

Estos argumentos, hacen incuestionable, la existencia de un riesgo a deshumanizar los cuidados de enfermería, ya que se describen actitudes y conductas que influyen en la aparición de este fenómeno, las cuales son contrarias a los principios éticos, morales y humanos que rigen la actuación profesional, conducta que surge, como consecuencia de los distintos avances tecnológicos, la desintegración de la práctica-docencia-investigacióninteracción social, el ascenso de tareas delegadas y la tecnicidad, la sumisión profesional y el desinterés por asumir una visión paradigmática que conciba el cuidado como el objeto de la profesión y no sea más que un mero concepto, como precisa Rodríguez (2011), en el que:

La gran cantidad de tareas que se encomiendan a diario a este tipo de profesional, le convierten en una especie de máquina que se le programa para producir trabajo en masa, olvidando el sentido humanístico de la atención; por la diversidad de labores encomendadas, el fin de la atención se enfoca en sacar cantidad, olvidando en muchas ocasiones la calidad de la tarea (págs. 39-40).

En este sentido, Subirana, Guillaumet, Fargues y Bros (2005), exponen 
que la teoría de Watson (1988): nace "como respuesta a una observación de los continuos cambios producidos en las organizaciones sanitarias, con un enfoque excesivamente médico, técnico y, a su vez, económico, que va en detrimento de los valores del cuidado y de la Enfermería" (págs. 30-31).

Finalmente, se considera que la práctica de enfermería debe realizarse, desde la conceptualización y concepción de los aspectos humanos, a partir de procesos que coadyuven a construir relaciones de ayuda y confraternidad entre humanos, estableciendo actitudes que tributen al trato amoroso y se sustente consistentemente en un sistema de valores humanos, que la enfermera y el enfermero deben cultivar a lo largo de su vida profesional, integrando los conocimientos científicos para guiar su quehacer, como se aprecia en la tabla 1, en la entrevista hecha a los profesionales de enfermería de los establecimientos del Sistema Público Nacional de Salud (SPNS) del Hospital Dr. Jesús Mata de Gregorio y el Hospital Dr. José María Vargas, Caracas, Venezuela.

Tabla 1. Análisis y resultados de la entrevista a profundidad: La práctica de la enfermería en ambientes hospitalarios en Venezuela.

La práctica de la enfermería en ambientes hospitalarios en Venezuela

Informante clave 1: La enfermería es una profesión humanista, ya que velamos por el bienestar físico, mental, espiritual y social de las personas que cuidamos diariamente... la práctica de enfermería es el quehacer o las destrezas y habilidades propias para brindar una atención humanizada, lo cual conocemos actualmente como los cuidados humanos que proporciona el profesional de enfermería por medio del conocimiento técnico-científico.

Informante clave 2: La práctica de enfermería se caracteriza por brindar atención humanizada, donde están inmersos los valores personales y profesionales, una práctica impregnada totalmente de humanismo... el profesional de enfermería posee valores humanos y compromiso ético, con las personas que atiende, sus familiares y con la profesión, estando esto implícito en el día a día. Ese compromiso es esencial para brindar cuidados humanos.

Informante clave 3: A pesar que existe un número importante de profesionales de enfermería que realizan una excelente labor asistencial, cargada de humanismo y valores, con ética y vocación de servicio; existen también colegas que no saben lo que significa la práctica humanista de enfermería, porque no brindan una atención humanizada hacia el otro, realizan sus funciones basándose 
en una acción esencialmente mecánica, que desintegra la esencia del cuidado humano y se aleja del conjunto de deberes, derechos, normas éticas y morales que rigen el accionar de la enfermería.

Informante clave 4: La esencia del cuidado humano y la atención de enfermería, ha venido desmejorando a tal punto que muchos enfermeros y enfermeras, maltratan verbalmente al paciente y sus familiares, lo llaman por el nombre de la cama, o más grave aún, por la enfermedad que padece. Delegan muchas funciones en los familiares de éstos, como es la realización del aseo personal, estar pendiente del cumplimiento del medicamento o revisar constantemente las vías periféricas. Es decir, que se observa una aplicación de valores contrarios a los establecidos en el código deontológico del año 2009. Esto sencillamente para mí, no es humano, ni nada que se parezca.

\section{Análisis}

Los entrevistados describen que la práctica de enfermería, admite a las personas como seres sociales, compuestos de una estructura física, espiritual, mental y social, producto de su composición orgánica; valiéndose de una actuación que se enmarque en la aplicación de valores humanos, además de las cualidades y virtudes que deben estar presentes en los profesionales, mediante la aplicación de cuidados humanizados, sustentándose en principios humanísticos, normas éticas y morales, actitud humana y vocación de servicio, en el trabajo diario de éstos.

En este sentido, basan su acción humana en el respeto a la vida de las personas y su dignidad humana, considerándolas como el elemento principal, el sujeto o razón de ser del proceso de cuidado humano, con el fin de lograr la satisfacción de las necesidades humanas reales o potenciales, sentidas, percibidas y expresadas, que interfirieren su funcionamiento normal, por lo tanto, la práctica de enfermería entiende y emplea el humanismo, como elemento filosófico y esencial del reencuentro del individuo con lo humano.

Fuente: El Autor (2019).

\section{Conclusiones}

Esta sección tiene como propósito fundamental, mostrar las reflexiones de la investigación, surgidas a partir de la contrastación de la teoría emergente, con la teoría formal, considerándose que la práctica de enfermería es humanista, producto de la interconexión de los componentes denominados: valores éticos, morales y principios bioéticos, el humanismo, la actitud, la vocación de servicio y el conocimiento.

Admitiendo que la práctica de enfermería parte de las experiencias de

los actores sociales, donde se concibe al hombre, como un sujeto cuya 
composición física, mental, espiritual y social, es producto de su estructura biológica, lo cual conlleva al respeto a la vida y a la dignidad de las personas, ya que el fin último del mismo, consiste en lograr la satisfacción de las necesidades reales y potenciales que interfieren el funcionamiento básico del organismo.

En este sentido se establece que, durante su formación académica, el enfermero y la enfermera, adquieren conocimientos, habilidades y experiencias profesionales centradas en la capacitación de un ser integral, moldeando aspectos humanos, éticos, vocacionales y actitudinales que garanticen un saber productivo, a través de una práctica centrada en procesos individualizados, acordes con las realidades sociales, orientando su actuación en el bienestar de la persona.

De igual forma se establece que la escuela tiene un importante desempeño en la formación de los enfermeros y las enfermeras, ya que tanto el humanismo, como la vocación de servicio son aspectos que deben ser forjados a través de las estrategias didácticas, de modo que este proceso los conlleve a asumir actitudes y conductas establecidas como preceptos en los fundamentos jurídicos de esta profesión.

Estas aseveraciones, ponen de manifiesto la relevancia del estudio e implicaciones para esta área del conocimiento, por lo que es importante para esta investigación, plantearse las siguientes interrogantes: ¿Se involucran los organismos del estado, relacionados directamente con la formación de profesionales de enfermería, en garantizar una formación continua en todas las áreas de esta ciencia?; ¿El docente de enfermería aplica una formación humanista, donde conciba la esencia filosófica de esta ciencia?; ¿La fragmentación de las funciones asistenciales, administrativas, docentes e investigativas, son producto de la deshumanización del cuidado?; ¿Es importante proseguir estudios avanzados en el área de enfermería, como sustento para investigar y establecer los parámetros del cuidado en el contexto 
hospitalario de Venezuela?

En consecuencia, estas surgen, producto de la inacabada indagación que se concibe en los estudios cualitativos, lo que sin duda será un aliciente para continuar investigando sobre la práctica de enfermería en el contexto hospitalario venezolano.

\section{Referencias}

Acosta, M., \& Acosta, M. (2019a,b). Epistemología del Pensamiento Pedagógico como Eje Transformador en la Praxis Educativa. Revista Scientific, 4(13), 346-362, e-ISSN: 2542-2987. [Artículo en línea]. Recuperado de: https://doi.org/10.29394/Scientific.issn.25422987.2019.4.13.19.346-362

Constitución de la República Bolivariana de Venezuela (2009). Gaceta Oficial de la República Bolivariana de Venezuela, N. 5.908, de fecha 15 febrero. Caracas, Venezuela: La Asamblea Nacional de la República Bolivariana de Venezuela.

Código Deontológico (2009a,b). Código Deontológico de los Profesionales de Enfermería de la República Bolivariana de Venezuela. Número 12, folio 70, tomo 62. Maracay, estado Aragua, Venezuela: IV Asamblea Nacional extraordinaria.

Guba, E., \& Lincoln, Y. (2000a,b). Paradigmas en Competencia en la investigación cualitativa. En Denman, C. y J. Haro (comps.), Por los rincones. Antología de métodos cualitativos en la investigación social. Hermosillo, Sonora, México: El Colegio de Sonora.

Guerrero-Ramírez, R., Meneses-La Riva, M., \& De La Cruz-Ruiz, M. (2015a,b). Cuidado humanizado de enfermería según la teoría de Jean Watson, servicio de medicina del Hospital Daniel Alcides Carrión. Lima- Callao, 2015. Revista Enfermeria Herediana, 9(2), 133-142, ISSN: 1998-5487. Recuperado de: 
https://doi.org/10.20453/renh.v9i2.3017

Ley de Ejercicio Profesional de la Enfermería (2005a,b,c). Gaceta Oficial de

la República Bolivariana de Venezuela N.* 38.263, fecha 1 de septiembre. Caracas, Venezuela: La Asamblea Nacional de la República Bolivariana de Venezuela.

Rodríguez, A., (2011). Enfermería ante la deshumanización: una necesidad por ser considerada desde la filosofía de Emmanuel Levinas. Enfermería en Costa Rica, 32(1), 37-42, ISSN: 1409-1992. Recuperado de: https://www.binasss.sa.cr/revistas/enfermeria/v32n1/art7.pdf

Rojas, B. (2007). Investigación cualitativa. Fundamentos y Praxis. Caracas, Venezuela: Fondo Editorial de la Universidad Pedagógica Experimental Libertador - FEDUPEL.

Strauss, A., \& Corbin, J. (2002a,b). Bases de La investigación cualitativa: técnicas y procedimientos para desarrollar la teoría fundamentada. Medellín, Colombia: Editorial Universidad de Antioquia.

Subirana, M., Guillaumet, M., Fargues, O., \& Bros, M. (2005). Teoría del cuidado humano: Un café con Watson. Metas de enfermería, 8(2), 28-32, ISSN: 1138-7262. España: Difusión Avances de Enfermería.

Taylor, S., \& Bogdan, R. (1984). Introducción a los métodos cualitativos de investigación. Barcelona, España: Ediciones Paidós.

Terán, C., \& Rico, Y. (2018). Mirada Axiológica al Discurso Formativo del Profesional de Enfermería. Revista Scientific, 3(9), 61-79, e-ISSN: 2542-2987. Recuperado de:

https://doi.org/10.29394/Scientific.issn.2542-2987.2018.3.9.3.61-79 


\section{Rubén Eliut Hernández Ortega}

e-mail: rubenho1977@gmail.com

Nacido en Güiria, estado Sucre, Venezuela, el 3 de

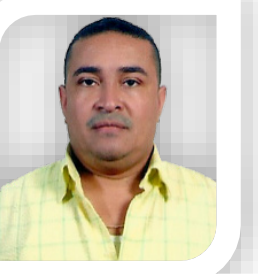
marzo del año 1977. Doctor en Ciencias de la Educación de la Universidad Latinoamericana y del Caribe (ULAC); Magister Scientiarum en Educación Superior de la Caribbean International University (CIU); Licenciado en Enfermería de la Universidad Nacional Experimental de los Llanos Centrales Rómulo Gallegos (UNERG); Director de los Programas Nacionales de Formación Avanzada en Enfermería de la Universidad de las Ciencias de la Salud (UCS), desde el año 2018 hasta la actualidad; Coordinador Docente de la Dirección Nacional de Enfermería del Ministerio del Poder Popular para la Salud (2016-2018); Coordinador del PNF de Enfermería del Distrito Capital (2012-2016); Docente del PNF de Enfermería de la Universidad Politécnica Territorial de los Altos Mirandinos Cecilio Acosta (UPTAMCA); Docente agregado con experiencia de 13 años; Enfermero con experiencia de 18 años; Miembro de la Línea de Investigación Proceso de cuidado integral en la salud mental de las personas, familias y comunidades; Miembro del comité de diseño curricular de los postgrados en enfermería de la Universidad de las Ciencias de la Salud (UCS); Autor de artículos arbitrados en la Revista Scientific (e-ISSN: 2542-2987); Tutor de trabajo de investigación en pregrado, trabajo especial de grado y tesis doctoral.

El contenido de este manuscrito se difunde bajo una Licencia de Creative Commons ReconocimientoNoComercial-Compartirlgual 4.0 Internacional 